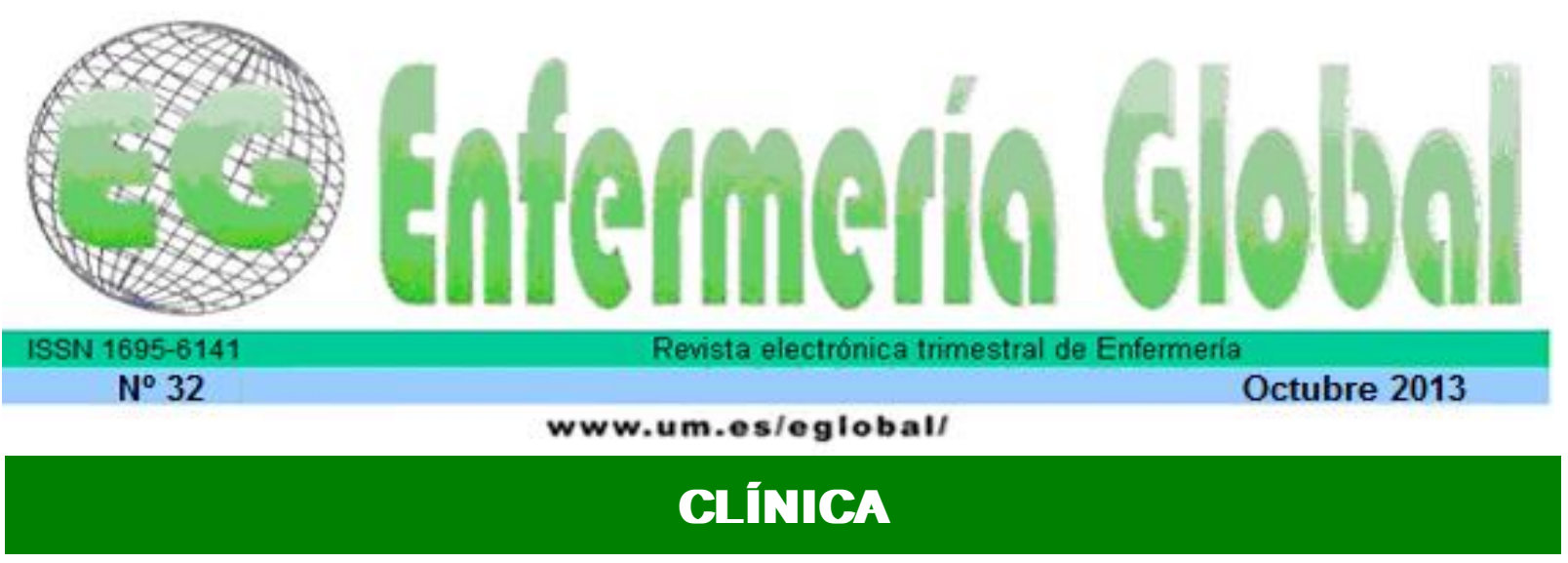

\title{
Ansiedad en gastroscopias: Comparación de dos intervenciones de enfermería en gastroscopias sin sedación
}

Anxiety in gastroscopies: Comparison of two nursing interventions in endoscopy without sedation

\section{*García Sierra, Rosa, "Caballero Sáez, Yolanda, *Mena Sánchez, Raquel}

\author{
*Enfermera Unidad de Endoscopias. Consorci Sanitari de Terrassa. Barcelona. E-mail: \\ rgarcias@cst.cat
}

Palabras clave: Gastroscopia; endoscopia; enfermería; intervenciones; ansiedad; tolerancia; satisfacción; investigación; quase-experimental;STAI

Keywords: Gastroscopy; endoscopy; Nursing Interventions; anxiety; tolerance; satisfaction; Investigation; Quasi-experimental; STAI.

\section{RESUMEN}

Existe disparidad en las percepciones que las enfermeras de endoscopias tenemos sobre la intervención de enfermería idónea en gastroscopias sin sedación. Algunas enfermeras piensan que el aporte de información es suficiente para reducir la ansiedad, mejorar la tolerancia y satisfacción, mientras otras defienden que además es necesario un entrenamiento conductual y un refuerzo positivo durante la prueba.

Los objetivos de esta investigación fueron comprobar las diferencias que se producían en el estado de ansiedad del paciente entre las dos intervenciones de enfermería, así como en la tolerancia y su satisfacción.

Se incluyeron 109 pacientes que acudieron vía ambulatoria a realizarse una gastroscopia sin sedación. Se dividieron en dos grupos, el experimental, con una intervención basada en información, entrenamiento conductual y refuerzo positivo durante la exploración y el control, con una intervención basada en la información. La ansiedad se evaluó con el test STAI-estado y con parámetros psicofísicos en diferentes momentos del proceso.

Los datos se analizaron con medidas repetidas de análisis de la variancia que aportaron los siguientes resultados: la puntuación del STAI disminuyó más en el grupo experimental $(p=0,035)$. La tolerancia fue mejor en el grupo experimental $(p=0,008)$, la satisfacción del paciente fue igual en los dos grupos $(p=0,5)$ la diferencia en los valores de tensión arterial sistólica, diastólica y frecuencia cardíaca fue igual en los dos grupos $(p=0,085, p=0,690, p=0,984)$

Las investigaciones experimentales son posibles en enfermería para obtener evidencias científicas sobre la idónea práctica clínica. Es posible mejorar la tolerancia de la gastroscopia y disminuir la 
ansiedad debida al procedimiento, con una intervención de enfermería centrada en el aspecto cognitivo y conductual de la persona.

\section{ABSTRACT}

There is a disparity between nurses about the ideal role of nurses in endoscopies without sedation. Some nurses think that providing information to the patient is sufficient to reduce anxiety and improve tolerance and satisfaction, while others believe that behavioral training and positive reinforcement during the procedure are also necessary.

The objectives of this study were to test the differences that are produced in the patient's state of anxiety between the two types of nursing intervention, as well as in the patient's tolerance and satisfaction.

The study included 109 outpatients who had an endoscopy without sedation. They were divided into two groups, the experimental group who received nursing support based on information, behavioral training and positive reinforcement during the procedure, and the control group, who received nursing support based solely on information provided about the procedure. Anxiety was evaluated with a STAI-state test and with psychophysiological parameters at different moments during the process. The data was analyzed with repeated measures of analysis of the variance, which resulted in the following: the STAI score decreased more in the experimental group. Tolerance was greater in the experimental group, patient satisfaction was equal in the two groups, and the difference in the levels of systolic and diastolic blood pressure and heart rate was equal in the two groups.

Experimental investigations are useful in nursing to obtain scientific evidence about the ideal clinical practice. It is possible to improve the tolerance of gastroscopy and reduce anxiety due to the procedure, with the intervention of the nurses centered in the cognitive and behavioral aspects of the person.

\section{INTRODUCCIÓN}

La endoscopia digestiva alta o gastroscopia se ha convertido en un procedimiento común, que se realiza con seguridad. En estos últimos años, el calibre del gastroscopio ha disminuido, mientras que la maniobrabilidad ha mejorado, es por tanto una técnica más fácil de realizar. Sin embargo no es un procedimiento cómodo para el paciente, que manifiesta ansiedad antes de la gastroscopia. Si eliminamos tales sentimientos de angustia, el paciente tiene más probabilidades de tolerar la gastroscopia sin problemas. Una adecuada intervención educativa, mejora la cooperación del paciente durante el procedimiento, disminuyendo la necesidad de repetir la gastroscopia y los costos consiguientes. ${ }^{1}$

La literatura refleja la preocupación por los estados de ansiedad pre-procedimiento en el ámbito quirúrgico ${ }^{2}$, y demuestra cómo la reducción de los niveles de ansiedad previos al procedimiento invasivo, puede facilitar la adaptación a los procedimientos y post-procedimiento. Algunas de las técnicas para reducir la ansiedad preoperatoria incluyen técnicas de relajación o de reestructuración cognitiva, que se demuestran efectivas ante procedimientos quirúrgicos ${ }^{3}$.

El tratamiento farmacológico puede conseguir un control adecuado de la ansiedad previa a la gastroscopia. No obstante, la literatura indica que la mayoría de las complicaciones durante el examen endoscópico se relacionan con la sedación ${ }^{4}$. Particularmente el riesgo de depresión cardiopulmonar ha sido constatada en pacientes de edad avanzada ${ }^{5,6}$. 
Otros estudios favorecen las medidas no invasivas reductoras de la ansiedad, y con efectos calmantes, tales como olores de lavanda 7 , la intervención musical ${ }^{8,9}$, y grabaciones de videos pacíficos relacionados con el medio ambiente natural, se asocian a la reducción de la ansiedad y a un mejor ambiente hospitalario ${ }^{10}$.

Por otra parte, la revisión bibliográfica refleja la importancia de la información sobre los procedimientos endoscópicos en la mejora de la aceptación de la prueba por parte del paciente. Se comparan diferentes maneras de aportar esta información, para finalizar que lo idóneo es acompañar la información verbal a la escrita, aunque se hace más hincapié en pruebas que potencialmente son más complicadas como es la colonoscopia ${ }^{11}$.

En relación a los estudios realizados resultado de la preocupación por la ansiedad en endoscopia digestiva ${ }^{12}$, éstos demuestran correlación entre el estado de ansiedad previo a la colonoscopia y la valoración del dolor de la misma. Ya concretamente en relación a la gastroscopia, destacamos el artículo publicado por Maguire, Walsh, y Little $^{13}$, en el que concluyen que la información previa a la gastroscopia es una medida efectiva para reducir la ansiedad así como la combinación de información y entrenamiento, aunque ésta última es comparativamente menos potente, resultado que no da soporte a la hipótesis inicial y que sorprende a los investigadores ${ }^{13}$.

Más recientemente, Hoya demuestra, cómo el ambiente calmado óptimo es reductor de la ansiedad previa a la gastroscopia ${ }^{14}$.

La búsqueda bibliográfica realizada en las bases de datos Medline y PsycINFO no proporcionó ninguna investigación que integre la valoración de los estados de ansiedad, la tolerancia observada por la enfermera y la satisfacción referida por el paciente.

El objetivo principal de la investigación fue comparar la ansiedad en pacientes que se realizaron una gastroscopia con la intervención de enfermería habitual, centrada en el aspecto cognitivo de la persona, con una intervención de enfermería centrada no solo en el aspecto cognitivo, sino también en el conductual.

Los objetivos específicos fueron comparar las dos intervenciones en los aspectos de tolerancia de la prueba y satisfacción del paciente.

La hipótesis que planteamos fue que una intervención protocolizada basada en el aporte de información temporal y sensorial, entrenamiento conductual y refuerzo positivo durante la exploración, disminuye la ansiedad y aumenta la tolerancia y satisfacción del paciente en mayor medida que una intervención centrada únicamente en la información.

\section{MATERIAL Y MÉTODO}

Diseño

Estudio cuasiexperimental. 


\section{Participantes}

El estudio fue aprobado por el Comité de Ética e Investigación Clínica del Consorcio Sanitario de Terrassa. 109 pacientes entre 18 y 85 años estuvieron de acuerdo en participar a través de la lectura de una hoja informativa y firma del consentimiento informado. La distribución de la muestra en el grupo experimental y el equivalente control, se realizó según conveniencia de la organización asistencial, asignándose la calidad de experimental al grupo de pacientes citados entre las 9 y las 14 horas y de grupo control a los pacientes citados entre las 15 y las 20 horas.

Fueron invitados a participar en la investigación todos los pacientes que acudían a realizar una gastroscopia diagnóstica sin sedación, vía ambulatoria que fueran mayores de edad, y que pasasen por el punto administrativo antes de llegar a la sala de espera de los gabinetes de exploración, entre las fechas 1 de Abril de 2011 y 30 de Junio de 2011, en la Unidad de Endoscopia Digestiva del CST. Fue criterio de exclusión que la persona no tuviera capacidad lectora para rellenar un autoregistro.

\section{Variables}

- Ansiedad: Spielberg State/Trait Anxiety Inventory (STAI)

El STAI es un cuestionario de 40 ítems de respuesta tipo Likert, que evalúa por separado la ansiedad rasgo (20 ítems) y la ansiedad estado (20 ítems). En esta investigación se utilizó la escala correspondiente a la ansiedad estado.

\section{- Frecuencia cardiaca}

Se utiliza para las medidas repetidas intrasujeto de frecuencia cardíaca un pulsioximetro marca NIHON KOHDEN, modelo KC-013P.

- Tensión arterial

Se utiliza para las medidas repetidas intrasujeto de tensión arterial un manguito marca NIHON KOHDEN, modelo KC-013P.

\section{- Tolerancia de la exploración}

La enfermera evaluó la tolerancia de la exploración a través de un formulario creado ad hoc en el que se registraron en escala Likert de 0 a 5 las nauseas, vómitos, verbalización de dolor e intentos de extracción del tubo, siendo 0 el mínimo valor posible y 5 el máximo. El rango de puntuación iba entre 0 a 20. (Figura 1)

Figura 1. Evaluación de la tolerancia realizada por la enfermera

\begin{tabular}{llllll}
\hline NÁUSEAS & 0 & 1 & 2 & 3 & 4 \\
\hline MOVIMIENTOS & 0 & 1 & 2 & 3 & 4 \\
\hline VERBALIZACIÓN DE DOLOR & 0 & 1 & 2 & 3 & 4 \\
\hline INTENTO EXTRACCION TUBO & 0 & 1 & 2 & 3 & 4
\end{tabular}


- Satisfacción

El usuario evaluó la satisfacción que sintió con la prueba contestando a un cuestionario creado ad hoc, con tres preguntas y tres opciones de respuesta. (Figura 2)

Figura 2. Satisfacción del paciente.

\section{Haga una cruz sobre la respuesta que indique mejor su satisfacción respecto a la prueba que acaba de realizarse.}

La explicación que le han dado sobre el procedimiento ha sido

Excelente Buena Mala

El malestar que ha sentido durante el procedimiento ha sido

\begin{tabular}{ccc}
\hline Insoportable & Tolerable & Ninguno \\
\hline ¿Volvería a repetir la prueba en las mismas condiciones? & Sí \\
\hline Nunca & Quizás & \\
\hline
\end{tabular}

\section{Procedimiento}

El paciente recibe a su llegada al punto administrativo de la unidad de endoscopias una hoja informativa del estudio y otra del consentimiento informado en la cual se le indica que debe firmar si acepta su participación. Junto con el consentimiento se entrega el autoregistro STAl-estado para que lo cumplimente mientras espera su turno para la realización de la prueba. La figura 3 facilita una visión global del procedimiento. 
Figura 3. Algoritmo del procedimiento

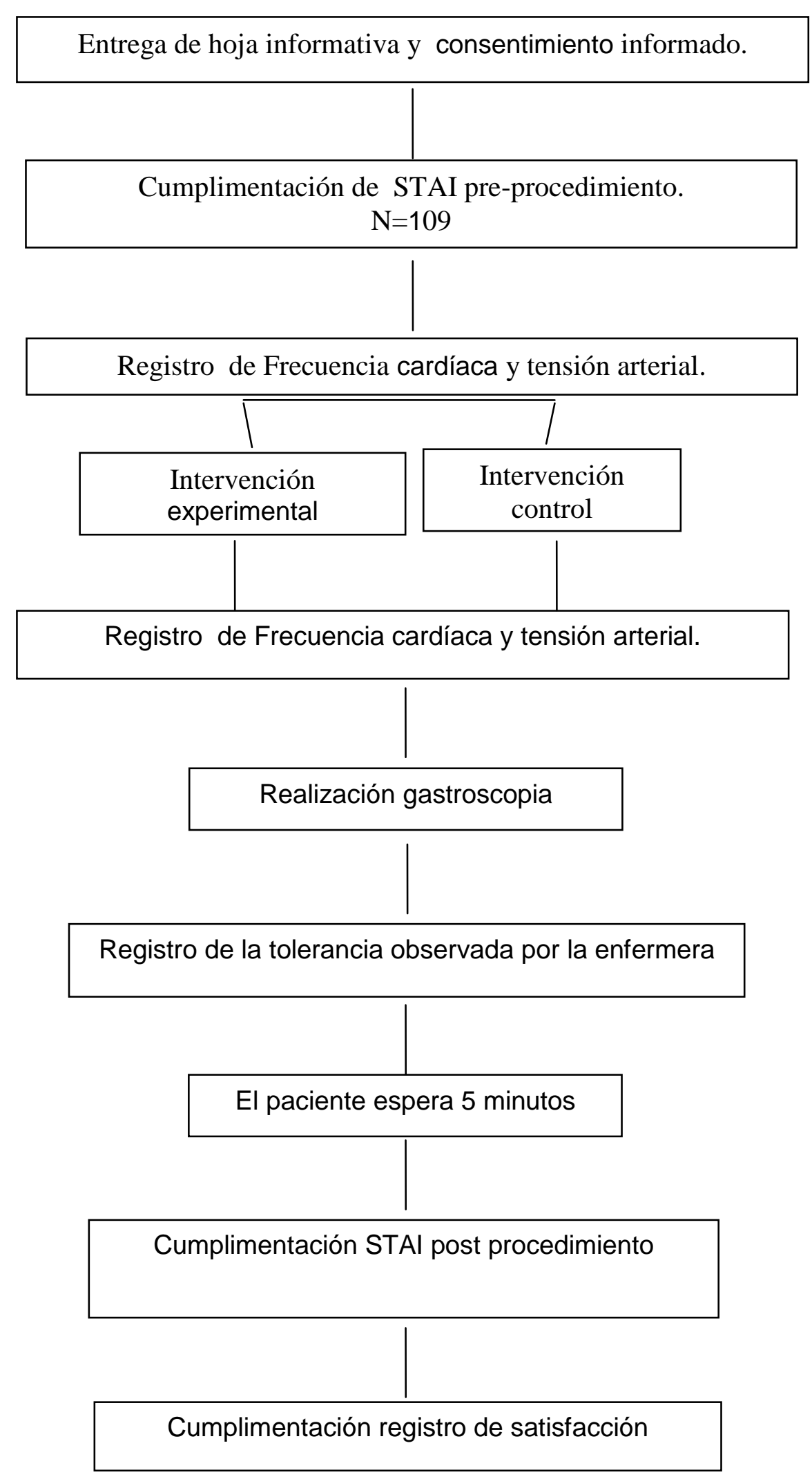

En el grupo experimental, la enfermera llama al paciente, realiza una toma de las constantes frecuencia cardíaca y tensión arterial del paciente y realiza la intervención de enfermería consistente en aportar información sensorial sobre el procedimiento, en la que se detalla lo que va a sentir durante la prueba (náuseas, sensación de plenitud 
gástrica, abundante salivación) y lo que puede hacer para contrarrestarlo (intentar no tragar la saliva, intentar retener el aire en el estómago,) y temporal especificando la duración aproximada de la prueba, entre tres y cuatro minutos, además, se realiza una intervención centrada en el aspecto conductual de la persona, consistente en el entrenamiento de habilidades como es la práctica de respiración profunda. Durante la exploración la enfermera realiza refuerzo positivo de todos los intentos del paciente por seguir las instrucciones.

El procedimiento seguido por el grupo control es el mismo, excepto la intervención de enfermería que consiste únicamente en el aporte de información sensorial y temporal previa a la exploración.

Después de la intervención de enfermería se realiza una nueva medida de las constantes frecuencia cardíaca y tensión arterial y se procede a la realización de la endoscopia. Una vez finalizado el procedimiento, la enfermera registra las observaciones que ha realizado sobre la tolerancia del paciente.

Cuando el paciente se ha recuperado de la exploración, aproximadamente unos 5 minutos después de retirar el endoscopio, procede a la cumplimentación del cuestionario STAI post procedimiento y del cuestionario de satisfacción.

Todas las gastroscopias que se han incluido en esta investigación han sido realizadas con gastroscopios Olympus, modelo EVIS EXERA II GIF-H 180.

Las enfermeras que han actuado en el grupo control y en el grupo experimental han sido siempre las mismas, 2 para cada grupo. Previamente al inicio del estudio se consensuó la intervención de enfermería.

Los digestólogos que han realizado las exploraciones han sido 6 adjuntos del servicio de digestología, los cuales han participado indistintamente en las exploraciones de los dos grupos.

\section{RESULTADOS}

Los datos fueron introducidos en una base datos Excel y analizados con el programa SPSS versión 17.

En primer lugar se compararon las características de los dos grupos de estudio, a través de T-test de muestras independientes para comparar la equivalencia de los dos grupos.

Para comparar el grupo experimental y el grupo control en todas las variables dependientes evaluadas: Frecuencia cardíaca, tensión arterial sistólica, tensión arterial diastólica, ansiedad estado, tolerancia y satisfacción se utilizaron medidas repetidas de análisis de la variancia (ANOVA)

\section{Características demográficas y resultados descriptivos}

De los 109 pacientes que participaron en la investigación, 66 fueron mujeres, representando un $60,6 \%$ de la muestra. El rango de edad de los participantes fue entre 18 y 85 años, con una media de 50.85 años y desviación estándar de 15.1 . 


\section{Análisis cuantitativo}

El análisis reveló equivalencia de los dos grupos en todas las variables. En la tabla 1 se muestra la descripción de la muestra.

Tabla 1. Equivalencia variables independientes

\begin{tabular}{lll}
\hline VARIABLE & $\begin{array}{l}\text { GRUPO } \\
\text { EXPERIMENTAL } \\
n=51\end{array}$ & $\begin{array}{l}\text { GRUPO } \\
\text { CONTROL } \\
n=58\end{array}$ \\
\hline $\begin{array}{l}\text { Edad (años) } \\
\text { M (SD) }\end{array}$ & $53,36(17)$ & $48,64(13)$ \\
$\begin{array}{l}\text { Sexo (\% de mujeres) } \\
\text { STAl pre-procedimiento (\%) } \\
\text { M (SD) }\end{array}$ & 64,7 & 56,9 \\
$\begin{array}{l}\text { Frecuencia cardíaca llegada } \\
\text { M (SD) }\end{array}$ & $44,6(18,5)$ & $36,3(16,9)$ \\
$\begin{array}{l}\text { Tensión arterial sistólica llegada } \\
\text { M (SD) }\end{array}$ & $77,1(15,3)$ & $80,5(14)$ \\
$\begin{array}{l}\text { Tensión arterial diastólica } \\
\text { llegada } \\
\text { M (SD) }\end{array}$ & $138,3(21)$ & $137(20)$ \\
\hline & $81,9(13,25)$ & $78,31(13)$ \\
\hline
\end{tabular}

Se llevaron a cabo repetidas medidas de análisis de la variancia (ANOVA) para comparar las diferencias en todos los parámetros medidos entre el grupo control y el experimental, desglosamos los resultados en cada una de las variables analizadas. La tabla 2 muestra un resumen del análisis.

Tabla 2.- Análisis de la Variancia

\begin{tabular}{lll}
\hline & $\mathrm{F}$ & Sig. \\
\hline Porcentaje de diferencia STAI * Tipo de intervención & 4,585 & 0,035 \\
Satisfacción * Tipo de intervención & 0,453 & 0,503 \\
Tolerancia * tipo de intervención & 7,206 & 0,008 \\
$\begin{array}{l}\text { Frecuencia cardiaca llegada- Frecuencia cardiaca inicio * } \\
\text { tipo de intervención }\end{array}$ & 0,000 & 0,984 \\
$\begin{array}{l}\text { Tensión arterial sistólica llegada- Tensión arterial } \\
\text { sistólica inicio * tipo de intervención }\end{array}$ & \\
$\begin{array}{l}\text { Tensión arterial diastólica llegada- Tensión arterial } \\
\text { diastólica inicio * tipo de intervención }\end{array}$ & 0,160 & 0,085 \\
\hline
\end{tabular}

Ansiedad

La ansiedad ha sido valorada con cuatro parámetros diferentes, el primero es la diferencia del test STAI estado a la llegada al hospital y a la salida del hospital, el segundo parámetro es la diferencia de frecuencia cardíaca a la llegada al hospital y el momento de inicio de la prueba, el tercero es la diferencia entre tensión arterial sistólica entre la llegada al hospital y el inicio de la prueba, y el último parámetro es la diferencia entre tensión arterial diastólica a la llegada de la prueba y al inicio de la 
prueba. De estos cuatro criterios, el porcentaje de diferencia en el valor del STAI entre el grupo experimental (media 12,78 \%, intervalo de confianza al $95 \%$ entre 0,95 y $10,05 \%$ ) y el grupo control (media 5,5\% intervalo de confianza entre 7,67 y 17,90\%) resulta significativa $(p=0,035)$. Las diferencias entre las medias de los dos grupos en el resto de parámetros medidos no resultan significativas. Destacar, sin embargo, que las medias de las diferencias de tensión son positivas, es decir, que la tensión disminuye desde la llegada al hospital hasta el inicio de la prueba, los valores de frecuencia cardiaca son negativos, o sea, que la frecuencia cardíaca aumenta desde la llegada al hospital hasta el inicio de la prueba.

\section{Satisfacción}

La media de satisfacción por la prueba realizada referida por el usuario, no muestra diferencia significativa entre los dos grupos, $(p=0.503)$

\section{Tolerancia}

La diferencia entre las medias de tolerancia percibida por la enfermera de endoscopias en los dos grupos se muestra como significativa $(p=0,008)$ siendo la media de tolerancia para el grupo experimental de 1,08 (IC: $0,65-1,50)$ y para el grupo control de 2,07 (IC: 1.48-2,66)

\section{DISCUSIÓN}

Los objetivos de este estudio fueron comparar la ansiedad, tolerancia y satisfacción en dos grupos de personas a las que se les realizó una gastroscopia diagnóstica sin sedación. Esta investigación surgió como necesidad de encontrar una evidencia científica que apoyase la percepción que las investigadoras tenían en la práctica asistencial diaria y que consistía en que una intervención de enfermería compuesta de preparación cognitiva y conductual era más eficaz en la disminución de la ansiedad del paciente que la intervención únicamente cognitiva, y que a su vez esta disminución de ansiedad, favorecía una mejor tolerancia de la exploración y finalizaba con una mejora de la satisfacción del paciente.

Los resultados obtenidos apoyan la hipótesis solo parcialmente. El porcentaje de disminución de ansiedad pre-post medida con el STAI resulta superior (estadísticamente significativo) en el grupo experimental, este hecho apoyaría la hipótesis de estudio, sin embargo, las diferencias en los parámetros psicofísicos de la ansiedad no resultan estadísticamente significativas, resultando diferencias muy parecidas en los dos grupos. Este último resultado es consistente con los obtenidos por Maguire en $2004^{13}$.

El no encontrar diferencias en los parámetros psicofísicos coincide con los resultados del estudio realizado por Hayes en $2003^{9}$, en el que se encontraban diferencias significativas pre-post en el resultado del STAI, sin embargo, no existían diferencias pre-post en los signos vitales.

Por otro lado, la tolerancia de la gastroscopia observada por la enfermera, sí se muestra diferente estadísticamente, destacando además que en ambos casos es una buena tolerancia, recordemos que en la escala utilizada, la mejor tolerancia posible tiene el valor cero y la peor tolerancia posible tiene el valor 20. En este estudio el grupo control obtiene una tolerancia 2 y el grupo experimental una tolerancia 1 , 
aunque son valores significativamente diferentes, en ambos casos son satisfactorios, es decir, que la percepción que tienen las enfermeras de cómo tolera el paciente la prueba en todos los casos es buena.

El tercer punto para cumplir nuestros objetivos era conocer la experiencia del paciente, su satisfacción, y fue medida con una encuesta creada con esta finalidad, en esta encuesta la puntuación 0 significa la peor experiencia posible y la puntuación 6 la mejor experiencia posible, los valores obtenidos en los dos grupos estuvieron alrededor de 4, lo cual nos invita a pensar que la experiencia de los pacientes es buena, y por tanto podríamos concluir que la percepción de la enfermera es concordante con la experiencia del paciente. Si bien este sería un punto para analizar en profundidad y con todas las posibles variables que pudieran ser confusoras de este resultado.

\section{CONCLUSIONES}

Las investigaciones experimentales son útiles en enfermería para obtener evidencias científicas sobre la práctica clínica idónea. Es posible mejorar la tolerancia de la gastroscopia y reducir la ansiedad debida al procedimiento con una intervención de enfermería centrada en aspectos cognitivos y conductuales del paciente.

\section{BIBLIOGRAFÍA}

1. Abuksis, G., Mor, M., Segal, N., Shemesh, I., Morad, I., Plaut, S., Weiss E., Sulkes J., Fraser G. \& Niv Y. A patient education program is cost-effective for preventing failure of endoscopic procedures in a gastroenterology department.

Am J Gastroenterol 2001;96: 1786-1790

2. Fincher, W., Shaw, J. \& Ramelet, AS. The effectiveness of a standardised preoperative preparation in reducing child and parent anxiety: a single-blind randomised controlled trial. J Clin Nurs.. 2012; 21(7-8): 946-955.

3. Stoddard, Jill A., White, Kamila S., Covino, Nicholas A., \& Strauss, Lisa. (2005). Impact of a brief intervention on patient anxiety prior to day surgery. Journal of Clinical Psychology in Medical Settings. 2005; 12 (2): 99-110.

4. Scheffer, GJ. Conscious sedation for endoscopic procedures. Neth J Med. 2004; 62 (1): $1-3$.

5. Christe, C., Janssens, JP., Armenian, B., Herrmann, F. \& Vogt, N. Midazolam sedation for upper gastrointestinal endoscopy in older persons: a randomized, doubleblind, placebo-controlled study. J Am Geriatr Soc. 2000; 48(11): 1398-1403.

6. Trevisani, L., Sartori, S., Gaudenzi, P., Gilli, G., Matarese, G., Gullini, S. \& Abbasciano $V$. Upper gastrointestinal endoscopy: Are preparatory intervention or conscious sedation effective? A randomized trial. World J Gastroenterol. 2004;10: 3313-3317.

7. Motomura, N., Sakurai, A. \& Yotsuya, Y. Reduction of mental stress with lavender odorant. Percept Mot Skills. 2001; 93(3): 713-718.

8. Gillen, E., Biley F, \& Allen D. Effects of music listening on adult patients' preprocedural state anxiety in hospital Int J Evid Based Healthc. 2008;6 (1): 24-29.

9. Hayes, A., Buffum, M., Lanier, E., Radahl, E., \& Sasso, C. A music intervention to reduce anxiety prior to gastrointestinal procedures. Gastroenterol Nurs. 2003; 26 (4): $145-149$. 
10. Wesa, KM. and Grimm RH. Jr. Recommendations and guidelines regarding the preferred research protocol for investigating the impact of an optimal healing environment on patients with hypertension. Journal of Alternative and Complementary Medicine. 2004; 10, Suppl 1: 245-250.

11. Coombes, JM., Steiner, J.F., Bekelman, DB, Prochazka, AV. \& Denberg, TD. Clinical Outcomes Associated with Attempts to Educate Patients about Lower Endoscopy: A Narrative Review. J Community Health. 2008; 33: 149-157.

12. Ylinen, ER., Vehviläinen-Julkunen, K. \& Pietilä, AM. Effects of patients' anxiety, previous pain experience and non-drug interventions on the pain experience during colonoscopy. J Clin Nurs.2009; 18(13): 1937-1944.

13. Maguire, D., Walsh, J.C. \& Little, C.L. The effect of information and behavioural training on endoscopy patients' clinical outcomes. Patient Educ Couns. 2004; 54: 6165.

14. Hoya, Y., Matsumura, I., Fujita, T. \& Yanaga K. The use of nonpharmacological interventions to reduce anxiety in patients undergoing gastroscopy in a setting with an optimal soothing environment. Gastroenterol Nurs. 2008; 31(6): 395-399. 